\title{
La petite fille à la peau noire et aux masques blancs: Le coeur à rire et à pleurer de Maryse Condé
}

\author{
Ching Selao
}

This article examines Maryse Condé's Le coeur à rire et à pleurer in the light of Franz Fanon's reflections on colonial mimesis. Condé recounts the life of a child in Guadeloupe who must deal with her parents' alienation and the weight of a painful past, a past that at first seems to her equally exotic and surrealist. The proposed reading explores the colonial imaginary of a little girl with black skin and white masks, by exploring her search for an identity which, much like the clothes she is forced to wear, never quite seem to fit, as well as three determining aspects of her relationship to others: her ambiguous relationship with her parents, the children's games that hint at the realities of the adult world, and finally the time spent in school, which make her aware of her own alienation.

Après plusieurs romans qui lui ont valu divers prix et une notoriété dans le monde francophone, Maryse Condé publie, en 1999, Le cour à rire et à pleurer dans lequel elle retrace les souvenirs de son enfance. Sans aucun doute le plus autobiographique de tous ses écrits, ce court texte n'en demeure pas moins une cuvre de fiction, comme l'annonce la formule oxymorique qui sert de sous-titre au livre, Contes vrais de mon enfance. Invitant le lecteur à lire ou peut-être plutôt à écouter ses histoires, Condé place en exergue une citation de Proust qui, à l'instar de son sous-titre, traduit un désir de retrouver les précieux moments de l'enfance tout en marquant une rupture, une difficulté d'offrir un portrait fidèle de ce monde. « Ce que l'intelligence nous rend sous le nom de passé n'est pas lui », écrit Proust dans Contre Sainte-Beuve. Le propos de cet article ne cherchera donc pas à établir la véracité de ses « contes », mais se penchera plutôt sur l'« aliénation » que vit une famille bourgeoise dans un pays colonisé, en l'occurrence la Guadeloupe, et de ses effets sur la vie de la petite Maryse Boucolon, " digne rejeton d'une famille où l'on pétait plus haut que ses fesses » (42), selon les dires de certains, qui se trouve confrontée aux difficultés de définir le complexe d'infériorité et de supériorité de ses parents.

\section{Les parents}

Le caur à rire et à pleurer est composé de dix-sept petits récits, chacun d'entre eux portant un titre fort révélateur, dont le premier présente la famille de Maryse et révèle, dès les premières lignes, l'amour de ses parents pour la France, une France non associée au pouvoir colonial mais considérée comme la véritable mère patrie. Pour ceux-ci, la Deuxième Guerre mondiale est sans conteste la période la plus difficile de leur vie, un moment tragique non pas à cause de l'extermination 
de six millions de Juifs ni de tous les autres crimes contre l'humanité, mais parce que cette interminable guerre les a privés, pendant sept longues années, de leurs voyages bien mérités en métropole. En introduisant ce raisonnement qui n'est pas sans étonner, voire choquer, Maryse souligne d'emblée l'admiration démesurée de ses parents pour qui chaque escapade en France représente autant de façons de prouver qu'ils sont français. Ainsi, après la fin de la guerre, la petite découvre, assise à une des terrasses du Quartier latin, l'attitude étrange de ses parents face aux paroles des garçons de café qui, croyant les complimenter, s'empressent de leur dire qu'ils parlent bien le français.

- Pourtant, nous sommes aussi français qu'eux, soupirait mon père.

- Plus français, renchérissait ma mère avec violence. [...] Nous sommes plus instruits. Nous avons de meilleures manières. Nous lisons davantage. Certains d'entre eux n'ont jamais quitté Paris alors que nous connaissons le Mont-Saint-Michel, la Côte d'Azur et la Côte basque. (13)

Pour l'esprit précoce de Maryse, cet épisode témoigne du paternalisme des garçons de table et met en évidence une injustice leur permettant de s'élever audessus de leurs clients noirs parce qu'« ils poss[èd]ent naturellement cette identité française » (13). Mais tandis que les justifications de sa mère tentent de rétablir leur « supériorité », elles ne font que créer chez la petite un malaise que celle-ci s'explique très mal: « Et moi, je ne comprenais pas en vertu de quoi ces gens orgueilleux, contents d'eux-mêmes, notables dans leur pays, rivalisaient avec les garçons qui les servaient » (14).

Afin de comprendre le comportement de ses parents, Maryse se tourne un jour vers son frère Alexandre, alias Sandrino, qui lui répond tout simplement: « T'occupe pas [...]. Papa et maman sont une paire d'aliénés » (15). Sans oser poser plus de questions et ne sachant toutefois pas ce que veut dire " aliénés ", Maryse finit par émettre elle-même sa propre théorie: « Une personne aliénée est une personne qui cherche à être ce qu'elle ne peut pas être parce qu'elle n'aime pas être ce qu'elle est » (16). Il apparaît fort intéressant de lire cette définition, articulée par une petite fille qui tente de saisir le monde complexe de ses parents, en parallèle avec l'analyse proposée par Frantz Fanon dans son fameux Peau noire, masques blancs ${ }^{\mathrm{i}}$. De fait, comme l'a si bien expliqué Fanon, la situation coloniale a créé, chez les Antillais - et chez les colonisés d'autres continents un complexe d'infériorité les amenant à vouloir être ce qu'ils ne sont pas, c'est-àdire blancs, puisqu'ils n'aiment pas ce qu'ils sont, en l'occurrence noirs. Suivant la logique fanonienne, la fascination des parents de Maryse pour la France s'expliquerait par le fait que « [1]e Noir qui connaît la métropole est un demi-dieu » (Fanon 1952, 15). Et pourtant, il semble qu'être un demi-dieu aux Antilles signifie très peu, sinon rien, une fois à Paris. Comme le montrent l'exaspération du père à qui l'on rappelle incessamment qu'il n'est pas français «Qu'est-ce que vous parlez bien le français! » (13), autrement dit: « Qu'est-ce que vous parlez bien le français pour une personne qui n'est pas française et, de surcroît, qui n'est pas blanche! »- et les justifications de la mère pour qui, de 
toute évidence, l'identité française est synonyme d'instruction et de culture, la difficulté n'est pas de vouloir être français, mais d'être perçue comme tel par les « vrais » Français.

S'il est clair que les parents de Maryse sont « aliénés » dans la mesure où ils aspirent à une identité qui leur est toujours niée, Leah Hewitt indique à juste titre que « if the chapter were to stop here, it would not quite be "Condéan" (sic), because the characterizations would be a bit too tidy, too definitive » (79). En effet, leur « aliénation " ne correspond pas tout à fait à celle décrite par Fanon dont l'argument premier repose sur le désir jamais assouvi du Noir d'être un Blanc, un désir qui n'est pas celui de ses parents et qui, par conséquent, n'est pas sans ajouter quelques difficultés quant à la compréhension de Maryse:

Mes parents étaient-ils des aliénés? Sûr et certain, ils n'éprouvaient aucun orgueil de leur héritage africain. Ils l'ignoraient. C'est un fait! [...] Comme ma mère, [mon père] était convaincu que seule la culture occidentale vaut la peine d'exister et il se montrait reconnaissant envers la France qui leur avait permis de l'obtenir. En même temps, ni l'un ni l'autre n'éprouvaient le moindre sentiment d'infériorité à cause de leur couleur. Ils se croyaient les plus brillants, les plus intelligents, la preuve par neuf de 1'avancement de leur Race de Grands-Nègres.

Est-ce cela être "aliéné"? (18-19)

Au sujet de cet extrait, Hewitt note le caractère complexe de la situation des parents en évitant toutefois de répondre à la question, concluant simplement que " $[\mathrm{t}]$ here is no unqualified answer to her question " $(80)$. Si la critique ne fait aucune référence à Fanon, l'écho n'en demeure pas moins évident. Il semble que Condé s'amuse à complexifier la thèse de ce dernier, tout en s'en inspirant fortement. Selon Fanon, le complexe d'infériorité est le résultat d'un double processus: « économique d'abord; par intériorisation ou, mieux, épidermisation de cette infériorité, ensuite » $(1952,8)$. Suivant cette définition, on pourrait rapidement déduire, en s'appuyant sur l'extrait cité ci-haut, que les parents de Maryse sont tout simplement européocentriques et que la couleur de la peau n'a rien à voir avec leur valorisation de la culture occidentale. Guadeloupéens bourgeois fiers d'appartenir à la « Race de Grands-Nègres » et ne parlant pourtant jamais le créole et interdisant leurs enfants de fréquenter les « petitsnègres " (les pauvres) de peur que les manières et le parler de ceux-ci ne les contaminent, il semble que ni l'aspect économique ni l'aspect épidermique ne les concernent. La mère accusera d'ailleurs Maryse d'un « crime » impardonnable lorsque cette dernière lui confiera qu'elle voit en Amélie Linsseuil, une jeune bourgeoise blanche, son idéal de beauté: « N'existait-il pas des personnes de couleur qui méritaient cette distinction? [...] S'ensuivit un discours bien senti dont les thèmes préfiguraient ceux de Black is beautiful » (93). Mais, pour la mère, " is Black really beautiful »? Rien n'est moins sûr. 
Alors que ses parents donnent l'impression qu'ils se prennent pour les plus brillants et les plus beaux, leur rejet de la culture guadeloupéenne, de même que leur refus systématique de parler le créole met pourtant en cause leur " fierté » d'être noirs. Fanon le souligne pertinemment: « le Noir sera d'autant plus blanc, c'est-à-dire se rapprochera d'autant plus du véritable homme, qu'il aura fait sienne la langue française " $(1952,14)$. En outre, une scène en particulier me paraît révélatrice d'une aliénation qui n'est certes pas étrangère à la couleur de la peau. Au retour d'une de ses escapades où Maryse s'est laissée porter par le vent plus longtemps qu'à son habitude, sa mère la réprimande: « Qu'est-ce que j'avais à courir comme une folle sous le soleil ? Est-ce que je n'étais pas assez laide et assez noire comme cela? » (135). La mère qui grondait sa petite pour avoir défini son idéal de beauté selon les traits d'une Blanche reproche ici à l'adolescente d'être laide et de l'être d'autant plus qu'elle est noire. Et pour marquer la noirceur de son enfant, elle ajoute que Maryse ressemble à une Kongo (135), chargeant celle-ci d'une faute: être aussi noire qu'une Africaine de l'Afrique noire. Qu'estil advenu de la mère qui discourait sur la beauté d'être noir(e)? Dans cette veine, on peut répondre par l'affirmative à la question de la narratrice, bien que l'aliénation de ses parents se présente du premier coup d'œil comme étant plus complexe et... moins « aliénante ».

\section{Jeux d'enfants}

Élevée dans un climat de colonisation mais choyée à outrance par sa famille aisée - même si la petite sait qu'elle n'a jamais été désirée puisque l'absence de menstruation avait fait croire à sa mère qu'elle vivait les premiers signes de sa ménopause - c'est à travers des « jeux d'enfants » que Maryse se retrouve pour la première fois confrontée à la charge historique de sa condition de Noire. Dans le chapitre intitulé " Leçon d'histoire ", la narratrice relate sa rencontre avec une fillette blanche qui l'interpelle en créole et lui propose de jouer avec elle à la condition de faire bien attention pour que sa mère ne les voit pas ensemble. D'abord insultée qu'on l'aborde en créole, elle qui appartient à une famille des plus respectables, mais se sentant si seule lors des interminables promenades de ses parents, Maryse se laisse rapidement entraîner dans des jeux qui ne lui plaisent guère, mais qui dureront tout de même plus d'une semaine:

Immédiatement, Anne-Marie prit la direction de nos jeux [...]. Je fus la mauvaise élève et elle me tira les cheveux. En plus, elle releva ma robe pour m'administrer la fessée. Je fus le cheval. Elle monta sur mon dos et elle me bourra les côtes de coups de pied. Je fus la bonne et elle me souffleta. Elle m'abreuvait de gros mots. (48)

Finalement lasse de tous ces sévices, Maryse décide un jour de dire à sa nouvelle " amie » de ne plus la frapper, ce à quoi Anne-Marie répond: « Je dois te donner des coups parce que tu es une négresse » (49). La fillette noire cherchera en vain la raison d'une telle affirmation. Sa mère évitera de répondre à sa question en lui demandant de cesser de raconter des bêtises puisque personne ne bat ni son papa 
ni sa maman. Son père, de son côté, lui répondra vaguement qu'on donnait des coups aux Noirs « dans le temps » (50), pour aussitôt la congédier. Ces jeux d'enfants révèlent donc quelque chose qui échappe à la petite, quelque chose qu'on persiste à lui cacher, que tous persistent à oublier:

Je devinais qu'un secret était caché au fond de mon passé, secret douloureux, secret honteux dont il aurait été inconvenant et peutêtre dangereux de forcer la connaissance. Il valait mieux l'enfouir au fin fond de ma mémoire comme mon père et ma mère, comme tous les gens que nous fréquentions, semblaient l'avoir fait. (51)

Dans cette atmosphère de déni où chacun tente d'oublier un passé trop lourd à porter, la narratrice - cette fois c'est l'adulte qui s'immisce dans la narration à l'instar de tous les autres habitants, efface à sa manière cet épisode en se questionnant sur sa vraisemblance: "Aujourd'hui, je me demande si cette rencontre ne fut pas surnaturelle. [...] Sinon comment expliquer ma docilité à moi si rebelle? » (51)

Puisque les adultes font tout pour ensevelir le passé et la mémoire, Maryse est amenée à faire face à la réalité de son existence et surtout de son apparence par le contact avec d'autres enfants. Dans le chapitre qui porte le titre du roman de Toni Morrison, The Bluest Eye, elle raconte l'échec de ce qui aurait pu être sa première histoire d'amour. Alors qu'elle nous prévient que Gilbert Driscoll est un mulâtre et qu'à cette époque, les Noirs, les Mulâtres et les Blancs ne se mélangeaient pas en Guadeloupe, elle ajoute qu' « [h]eureusement, les enfants ne s'occupaient pas tellement de ces affaires de grandes personnes " (62). Ici, tout porte à croire que les questions raciales ne concernent pas vraiment les enfants, que ceux-ci sont, en quelque sorte, encore "protégés " de l'univers des exclusions. Pourtant, lorsque Gilbert lui envoie une lettre, après des heures et des jours de regards d'adoration réciproques, le choc oblige Maryse à constater que l'aliénation les a déjà atteints. Les premiers mots de la missive sont éloquents et on aura compris la signification du titre qu'emprunte Condé à Morrison: " "Maryse adorée, pour moi, tu es la plus belle avec tes yeux bleus" " (65). Contrainte de se regarder dans la glace et de se voir telle qu'elle est, c'est-à-dire avec des yeux marron foncé, presque noirs, donc, rien à voir avec la personne à qui s'adressent ces quelques lignes, Maryse prend conscience du sentiment d'étrangeté imposé par le regard de l'autre. Or, en envoyant à Gilbert un petit mot dramatique annonçant leur " rupture " - " "Gilbert, tout est fini entre nous" » (66), courte phrase tirée d'une des lectures de sa sœur -, la petite fille répète elle aussi le mimétisme textuel de Gilbert qui avait copié les quelques lignes de "romans français de quatre sous » (66) ne correspondant aucunement à leur réalité. Selon Mireille Rosello, la missive de la fillette traduit sa force dans la mesure où celle-ci ne se laisse pas convaincre que, n'ayant pas les yeux bleus, elle n'est pas la plus belle: "Elle pourrait sombrer dans l'aliénation, la folie et le mépris d'elle-même. Mais l'héroïne des "contes vrais" réagit très différemment. [...] Elle rompt, instantanément, avec le maladroit 
épistolier [...]. Elle est capable, semble-t-il, de dire non et de mettre un point final à cette mascarade de blanchissement » (173). Mais si Maryse fait effectivement montre d'assurance, voire de courage en disant non, la fin de son " aventure » avec Gilbert met-elle vraiment un point final à « cette mascarade de blanchissement »?

\section{L'école}

L'épisode que raconte Maryse concernant son séjour en France alors qu'elle a environ treize ans semble plutôt indiquer que la mascarade n'est pas terminée et ce, bien que l'enfant prenne conscience de la situation singulière dans laquelle elle se retrouve. En effet, suite à la lecture du roman de l'auteur martiniquais Joseph Zobel, La Rue Cases-Nègres, Maryse découvre, à travers 1'histoire de José Hassan, la misère des Noirs, l'esclavage, l'oppression coloniale, l'univers de la plantation, l'exploitation de l'homme par l'homme: autant d'inégalités qui lui font réaliser que le milieu bourgeois auquel elle appartenait a fait d'elle « un mauvais décalque des petits Français qu['elle] côtoyai[t] » (120). De ce roman, lu en vue d'une présentation en classe sur un livre de « son » pays que lui propose de faire Mlle Lemarchand, sa professeure de français du lycée Fénelon ${ }^{\mathrm{ii}}$, Maryse admet que les injustices dont il est question lui sont complètement étrangères: « Pour moi, toute cette histoire était parfaitement exotique, surréaliste » (118). Mal à l'aise, toutefois, à l'idée de dévoiler qu'elle est coupable de ne pas être une " vraie Antillaise " comme l'est le personnage de Zobel puisqu'elle n'a pas connu les malheurs de José, la petite devient, malgré elle, l'Antillaise de service: « Je commençai par me révolter en pensant que l'identité est comme un vêtement qu'il faut enfiler bon gré, mal gré, qu'il vous siée ou non. Puis, je cédai à la pression et enfilai la défroque qui m'était offerte »(119). Ainsi, Maryse se retrouve dans une situation de double contrainte: d'une part, ses parents lui imposent une identification aux Français qui aboutit à un échec une fois en France et, d'autre part, les Français l'obligent à porter une identité qui ne correspond pas du tout à qui elle est.

Sa posture de l'entre-deux traduit 1'expression d'Homi Bhabha qui, à la suite de Fanon, parle du mimétisme colonial en ces termes fort révélateurs, « Not quite/ not White » (92), insistant dès lors sur l'importance de la couleur de la peau dans la construction des identités par le discours colonialiii. De fait, malgré son éducation à la française et sa méconnaissance de la culture et de l'histoire de la Guadeloupe, en particulier, et des Antilles, en général, la petite se voit contrainte de porter le vêtement que l'on associe à la couleur de sa peau, un vêtement qui doit trahir la pauvreté et la misère: « Depuis des jours, mon ventre traversé des gargouillis de la faim s'était ballonné. Mes jambes s'étaient arquées. Mon nez s'était empli de morve. La tignasse grenée de mes cheveux s'était roussie sur ma tête sous 1'effet du soleil. J'étais devenue Josélita, sœur ou cousine de mon héros » (120). Ce renvoi à sa 《 race » et cette identification forcée à un personnage imaginaire lui font néanmoins prendre conscience de son « aliénation », une aliénation dont elle tente de se distancier en affirmant, sur un ton plus ironique que dramatique: « J'étais "peau noire, masque blanc" et c'est 
pour moi que Frantz Fanon allait écrire » (120). Pourtant, être consciente de sa situation n'arrête nullement le regard de l'Autre, un regard qui l'exotise et la voit comme un spectacle ethnographique. Tandis qu'elle se met en scène devant ses camarades de classe, elle devient, pour les étrangers de la rue et du métro, un objet d'observation qui étonne:

- Elle est mignonne, la petite négresse!

Ce n'était pas le mot "négresse" qui me brûlait. En ce temps-là, il était usuel. C'était le ton. Surprise. J'étais une surprise. L'exception d'une race que les Blancs s'obstinaient à croire repoussante et barbare. (114)

Cet extrait n'est pas sans faire écho, à mon sens, au passage connu de Peau noire, masques blancs dans lequel Fanon dénonce le regard de la femme blanche sur lui: « - Regarde, il est beau, ce nègre... », ce à quoi il répond: " - Le beau nègre vous emmerde, madame! » $(1952,92)$. Si la réplique de Fanon est un signe de sa subjectivité, à l'intérieur même de son objectivité, comme le note Gwen Bergner: « By speaking of himself in the third person, Fanon derisively mimics the white woman's refusal to address him as a subject. He asserts his subjectivity even as he acknowledges his place as object of the gaze » (80); la jeune Maryse, quant à elle, ne répond pas à ces remarques et demeure donc dans un statut d'objet.

Dans Le cour à rire et à pleurer, l'école, plus que tout autre lieu, est l'endroit où Maryse ressent avec intensité son sentiment d'étrangeté, sentiment d'être déplacée que ne lui épargnent pas ses professeurs: "Les professeurs s'accordaient pour ne pas déranger ma paresse: leur attitude signifiait que cette petite Guadeloupéenne n'était pas à sa place ici, pas une candidate pour les grandes écoles » (141). En outre, si Mlle Lemarchand était pleine de bonnes intentions, Mme Épée - qui porte bien son nom tant elle prend plaisir à piquer Maryse - profite de son autorité pour tourner la classe en safari: « Désormais, les classes de français devinrent des zoos où un gardien exhibait des bêtes captives. Des arènes où le montreur les forçait à des tours » (141). De toute évidence, les étudiants africains et antillais de la classe forment un spectacle exotique pour l'enseignante. La métaphore du zoo met évidemment l'accent sur l'importance du regard imposé par Mme Épée, mais indique également le sentiment dégradant des étudiants. En les comparant à des « bêtes captives », la narratrice, qui n'est certes pas exclue de ce cirque, souligne l'attitude de Mme Épée qui, visiblement, les animalise. Si Fanon, dans Les damnées de la terre, affirme que « le langage du colon, quand il parle du colonisé, est un langage zoologique » $(1991,73)$, il apparaît que le comportement de cette maîtresse qu'il faut certainement entendre dans les deux sens de ce terme - ne se borne pas au langage, mais s'étend à une attitude des plus discriminatoires qui traduit non seulement un désir de chosifier ses élèves en les exhibant comme des objets exotiques, mais une volonté de les réduire à un état bestial. Dans ces « jeux du cirque » (142), Mme Épée impose à Maryse un rôle fixe, celui d'incarner la déchéance de l'Afrique déplacée de son continent, c'est-à-dire transposée dans le 
Nouveau Monde. Se permettant de constamment faire des comparaisons entre Maryse et Marguerite, une étudiante sénégalaise qui représente à ses yeux l'Afrique « pure », l'enseignante adopte un comportement colonialiste qui n'est pas dénué de contradictions:

Sans le savoir peut-être, [Mme Épée] rejoignait la longue lignée des missionnaires et des administrateurs coloniaux qui ont ridiculisé, vilipendé "l'Africain détribalisé", le "trousered nigger" sans vouloir reconnaître que Marguerite, formée dans un pensionnat catholique de Dakar, admise dans un des meilleurs lycées de Paris, n'était pas plus "pure" que moi. (142)

Marguerite elle-même confiera plus tard à Maryse que Mme Épée est une raciste qui déteste autant les Africains que les Antillais et que sa manière de diviser les groupes s'inscrit exactement dans le projet colonial qui lui permet de mieux régner.

Si ce cirque est dénoncé par plusieurs élèves de la classe de multiples façons qui manifestent par ailleurs une vive sympathie pour Maryse, celle-ci ne tarde toutefois pas à constater que ses amies tentent, elles aussi et à leurs manières, de l'enfermer dans les stéréotypes les plus contraignants. Ainsi, après chaque séjour à la maison de campagne des parents de ces nouvelles amies, Maryse remarque qu'elle n'échappe pas au rôle qu'on lui a déjà assigné:

Pourtant, de retour au foyer, j'avais à chaque fois conscience d'avoir tenu le rôle de la négresse à talents. Non, je ne sortais pas d'un champ de cannes. Oui, mes parents étaient des notables. Oui, j'avais toujours parlé le français dans ma famille. Mes camarades auraient aussi voulu que je me révolte, que je réponde aux attaques de mon assaillante. (142-143)

Or, dans cet univers de la jeune bourgeoisie antillaise dont fait pourtant partie Maryse, comment expliquer qu'on puisse la prendre pour un coupeur de cannes,

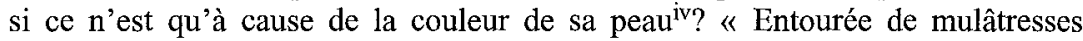
blondes ou brunes, bouclées ou frisottées, de chabines dorées aux yeux multicolores, verts, gris ou bleus, j'étais la seule à porter peau noire et cheveux grenés » (138), écrit la narratrice. Mais dans ce monde hybride où ces filles parlent le créole, ne se gênent pas pour rire très fort ou pour se laisser aller au rythme des biguines, Maryse semble être la seule à se contrôler, à adopter un comportement " convenable », ce qui fait dire à l'adolescente à la peau noire et aux masques blancs que ces jeunes filles sont plus près des « petits-nègres » qu'elle ne peut l'être, et ce en dépit de la couleur de leur peau:

Bien que ma couleur m'assimilât aux petits-nègres, coupeurs de cannes, ammareuses, pêcheurs, revendeuses, manœuvres sur les 
quais, que sais-je encore? j'étais plus éloignée d'eux que les donzelles à peau claire qui m'entouraient. [...] À croire que leurs parents ne leur avaient pas inculqué les bonnes manières! À croire qu'ils ne partageaient pas le mépris des miens pour les traditions locales! (139)

Même loin de ses parents, Maryse admet être demeurée leur fille, c'est-à-dire la petite qui, sans rêver d'être blanche puisqu'elle n'est pas sans ignorer l'impossibilité d'accéder à ce monde que lui a déjà interdit sa mère lorsqu'elle a osé voir en une femme blanche son idéal de beauté et que lui interdisent chaque jour les Français, reproduit néanmoins la mascarade de ses aînés qui consiste à se croire supérieurs aux autres Noirs et à considérer la culture antillaise et la langue créole comme des signes témoignant d'un manque de civilité.

En mettant en scène une petite fille bourgeoise à la peau noire, fière de sa peau noire comme le lui ont appris ses parents, et qui a pourtant recours aux masques blancs, Condé complexifie la théorie fanonienne qui explique le mimétisme colonial comme étant le résultat d'un complexe d'abord et avant tout économique et épidermique. Si Maryse et ses parents n'ont pas honte de leur peau noire, ils n'en rejettent pas moins les éléments culturels et langagiers qui sont associés à la couleur de cette peau. Dès lors, une question s'impose: leur fierté d'appartenir à la « Race de Grands-Nègres 》 (19) s'inscrit-elle dans un processus de désaliénation ou, au contraire, est-elle une preuve de leur aliénation, comme semble le suggérer Sandrino? Au terme du récit, Maryse revient notamment sur l'affirmation de son frère en précisant qu'elle touche sans doute au cœur du problème de ses parents. Ceux-ci n'étant jamais naturels (127), ils se maîtrisent constamment afin d'éviter que le surgissement soudain de ce qui serait profondément tapi au fond d'eux ne se produise. Cette retenue des parents que Maryse reconnaît comme n'étant pas « naturelle » n'est pas sans rappeler, par ailleurs, son propre contrôle devant les autres étudiantes antillaises envers lesquelles elle porte un jugement très sévère. Sans le vouloir, elle reproduit le comportement qu'enfant elle trouvait des plus étranges. Ainsi, une partie de l'héritage familial de Maryse aura été cette "mascarade ", ce mimétisme colonial qui fait que l'enfant, par une identification aux Français et ce malgré son éducation « noiriste » (152), devient la fille à la peau noire et aux masques blancs dont le miroir de la société ne lui renvoie pas le reflet espéré. En somme, Maryse est toujours en quête d'une identité inaccessible ou, pour le dire en ses termes, toujours à la recherche d'un vêtement qui l'habillerait pour qu'elle n'ait plus à porter ces masques.

\section{Notes}

${ }^{\text {i }}$ Il s'agit ici de lire Le coeur à rire et à pleurer en parallèle avec les propos de Fanon et non la vie de Condé, l'écrivaine et la critique. Cet article n'entend pas montrer les divergences d'opinions qui existent entre Fanon et Condé. Dans le film 
d'Isaac Julien, Frantz Fanon: Peau noire, masque blanc, Françoise Vergès note que Condé a fortement critiqué la lecture de Fanon de Je suis Martiniquaise de Mayotte Capécia. Condé elle-même prend la parole dans ce film en soulignant la vision réductrice de Fanon pour qui Mayotte Capécia représente l'ensemble des femmes noires (Julien). Par ailleurs, il importe de mentionner que la position de Condé par rapport à la « Négritude » est similaire à celle de Fanon. Alors que celui-ci écrit: « Le nègre n'est pas. Pas plus que le Blanc » (Fanon 1952, 187), celle-là, en réaction à la glorification du Noir par Aimé Césaire, répond: " le Nègre n'existe pas » (Condé 1974, 413), pour finalement conclure avec cette phrase qui abonde dans le sens de Fanon: " Notre libération passe par la connaissance qu'il n'y a jamais eu de Nègres. Il n'y a jamais eu que des hommes exploités » (Condé 1974, 419).

ii On notera que Zobel est Martiniquais et non Guadeloupéen, ce que Maryse explique comme suit: " Mais la Martinique est l'île sœur de la Guadeloupe » (117). Outre ladite parenté de ses deux îles, doit-on ici comprendre, implicitement, que de toute façon pour l'audience à qui s'adressera l'exposé, les Antilles ne forment qu'un tout homogène?

iii Homi Bhabha, s'inspirant de Fanon, souligne que le mimétisme est encouragé dans toute situation coloniale et constitue une stratégie de contrôle et de surveillance, car malgré le désir du colonisé de ressembler au colonisateur, $s a$ différence est là pour lui rappeler qu'il demeure l'autre reconnaissable, le colonisé étant « a subject of a difference that is almost the same, but not quite » (86). Le critique post-colonial insiste sur l'ambivalence du discours colonial qui encourage l'identification du colonisé au colonisateur pour ensuite le renvoyer à « sa» place. En termes fanoniens, le mimétisme donne naissance aux « damnés de la terre $»$ : "Pas encore blanc, plus tout à fait noir, j'étais un damné », écrit Fanon (1952, 112).

iv Dans le film d'Isaac Julien, Frantz Fanon: Peau noire, masque blanc, Maryse Condé confie ceci: « Quand j'ai lu "Peau Noire, Masques Blancs" j'étais au foyer étudiant des jeunes filles de ce qu'on peut appeler la bourgeoisie antillaise. Beaucoup d'entre nous étaient acceptées dans des grandes écoles de Paris. Nous avions l'impression que nous étions les plus intelligentes, les plus belles, les plus douées, que nous avions un avenir extraordinaire. Donc il était impossible pour nous de nous reconnaître dans les portraits que Frantz Fanon donnait des Antillais: des gens pathologiquement aliénés, pleins de complexes d'infériorité et qui étaient incapables d'assumer leur race. Donc la réaction que nous avons eue - je dis nous parce qu'il y avait tout un groupe de gens, autour de moi, des filles surtout - nous avons donc senti que ce livre-là ne représentait pas du tout notre moi collectif » (Julien). Cependant, il est clair que l'univers de Maryse dans Le cour à rire et à pleurer n'est pas étranger à celui décrit par Fanon. 


\section{Bibliographie}

Bergner, Gwen. « Who Is That Masked Woman ? or, The Role of Gender in Fanon's Black Skin, White Masks ». PMLA 110.1 (1995): 75-88.

Bhabha, Homi. The Location of Culture. New York: Routledge, 1994.

Condé, Maryse. Le coeur à rire et à pleurer. Contes vrais de mon enfance. Paris: Robert Laffont [Pocket], 1999.

— « Négritude césairienne, négritude senghorienne ». Revue de littérature comparée 48 (1974): 409-419.

Fanon, Frantz. Peau noire, masques blancs. Paris: Seuil, 1952. Les damnés de la terre. Paris: Gallimard, 1991 [1961].

Julien, Isaac. "Frantz Fanon: Peau noire, masque blanc", www.k-films.com/distribution/films/fanon/fanonscenar.html.

Hewitt, Leah. "Transmigrations in Maryse Condé's True Tales ». In French Prose in 2000. Michael Bishop and Christopher Elson (dir.). New York: Rodopi, 2002. 75-82.

Rosello, Mireille. " Le petit cornac des mots d'amour: Tragi-comédie en un adjectif ». In Maryse Condé: Une nomade inconvenante. Madeleine CottenetHage et Lydie Moudileno (dir.). Paris: Ibis Rouge, 2002. 169-174. 